\title{
Diffusion of Nanoparticles with Activated Hopping in Crowded Polymer Solutions
}

\author{
Chundong Xue, Xinghua Shi, Yu Tian, Xu Zheng,* and Guoqing $\mathrm{Hu}^{*}$ \\ Cite This: Nano Lett. 2020, 20, 3895-3904 \\ Read Online
}

ABSTRACT: A long-distance hop of diffusive nanoparticles (NPs) in crowded environments was commonly considered unlikely, and its characteristics remain unclear. In this work, we experimentally identify the occurrence of the intermittent hops of large NPs in crowded entangled poly(ethylene oxide) (PEO) solutions, which are attributed to thermally induced activated hopping. We show that the diffusion of NPs in crowded solutions is considered as a superposition of the activated hopping and the reptation of the polymer solution. Such activated hopping becomes significant when either the PEO molecular weight is large enough or the NP size is relatively small. We reveal that the time-dependent non-Gaussianity of the NP diffusion is determined by the competition of the short-time relaxation of a polymer entanglement strand, the activated hopping, and the long-time reptation. We propose an exponential scaling law $\tau_{\text {hop }} / \tau_{\mathrm{e}} \sim \exp \left(d / d_{\mathrm{t}}\right)$ to characterize the hopping time scale, suggesting a linear dependence of the activated hopping energy barrier on the dimensionless NP size. The activated hopping motion can only be observed between the onset time scale of the short-time relaxation of local entanglement strands and the termination time scale of the long-time relaxation. Our findings on activated hopping provide new insights into long-distance transportation of NPs in crowded biological environments, which is essential to the delivery and targeting of nanomedicines.

KEYWORDS: hopping diffusion, non-Gaussianity, activated energy barrier, crowded polymer solutions

\section{INTRODUCTION}

The diffusive motion of nanoparticles (NPs) in heterogeneous environments is intriguing as it exhibits the mesoscopic behavior that is distinct from the classical Brownian motion. Examples are abundant in polymer solutions, ${ }^{1-3}$ porous media, ${ }^{4-8}$ and especially biomacromolecular environments. ${ }^{9-14}$ Understanding the transport mechanism of NPs in these confinements has become a great challenge, which is also of crucial importance to the improved permeability and targeting of nanomedicines and various basic biological processes. ${ }^{15-17}$ Previous studies have demonstrated that the diffusion of NPs in complex fluids is influenced by the intrinsic structure of the surrounding medium. ${ }^{1,11,18}$ At short times, the NPs' motion is restricted by the surrounding structures, manifesting a sublinear mean square displacement (MSD), while at long times, the MSD is seemingly Fickian. The recently reported "anomalous yet Fickian" phenomenon further indicated the unusual dynamics of NPs in dilute polymer solutions, ${ }^{19,20}$ in which the non-Gaussianity in the displacement probability distribution (DPD) was observed, despite the simultaneous appearance of the linear MSD. This non-Gaussian behavior in dilute solutions has been connected to the regular hopping diffusion that occurs when the size of a NP is smaller than the typical mesh size of the polymer networks. ${ }^{19-21}$ It was well accepted that the NP can directly diffuse through the adjacent mesh and exhibit larger displacements and a non-Gaussian DPD. Therefore, in dilute solutions, this regular hopping diffusion has been deemed as the dominant mechanism that leads to the non-Gaussian anomaly. ${ }^{19,21,22}$

However, whether the mechanism of the hopping diffusion in dilute solutions can be applied to more crowded media is unclear. ${ }^{18,20,23}$ A long-distance hop of NPs in crowded media was thought to be unlikely because the typical mesh size of the crowded environments becomes much smaller than the NP size. Yet, such phenomenon, often referred to as a "rare event", has been observed in some crowded biological environments. ${ }^{24,25}$ This long-distance hop may play an essential role in many biological processes, ${ }^{16,18,19}$ while its physical origin needs to be clarified. The above-mentioned regular hopping diffusion in dilute solutions cannot explain the long-distance hops in crowded solutions because of the smaller mesh size and the stronger confinement. A new possible mechanism for a NP

Received: March 11, 2020

Published: March 25, 2020 
A

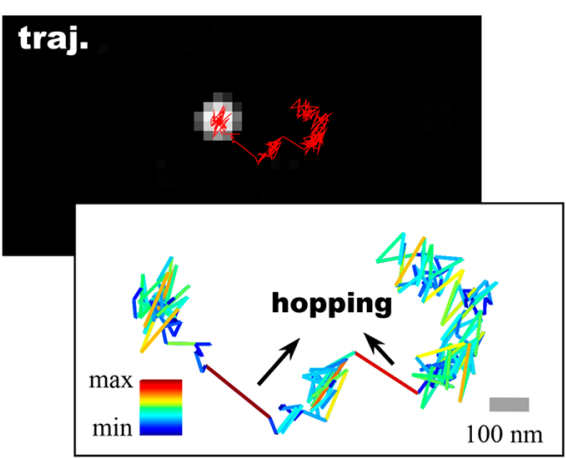

B

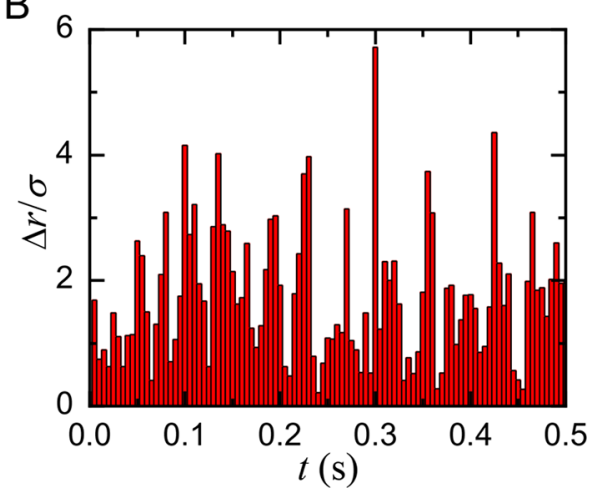

Figure 1. Particle tracking experiment and results. (A) An experimental image (top) of the typical trajectory (red line) of a NP ( $d=200 \mathrm{~nm})$ in a 0.8 wt \% (20 $*$ ) 8 M PEO solution. This trajectory is illustrated by the color-coded speed with a $5 \mathrm{~ms}$ time step (bottom). The color bar from blue to red represents the speed from 1 to $29 \mu \mathrm{m} / \mathrm{s}$. (B) The intermittent hops are highlighted by large displacements $\Delta r / \sigma$, where $\sigma$ is the standard deviation of the displacements.
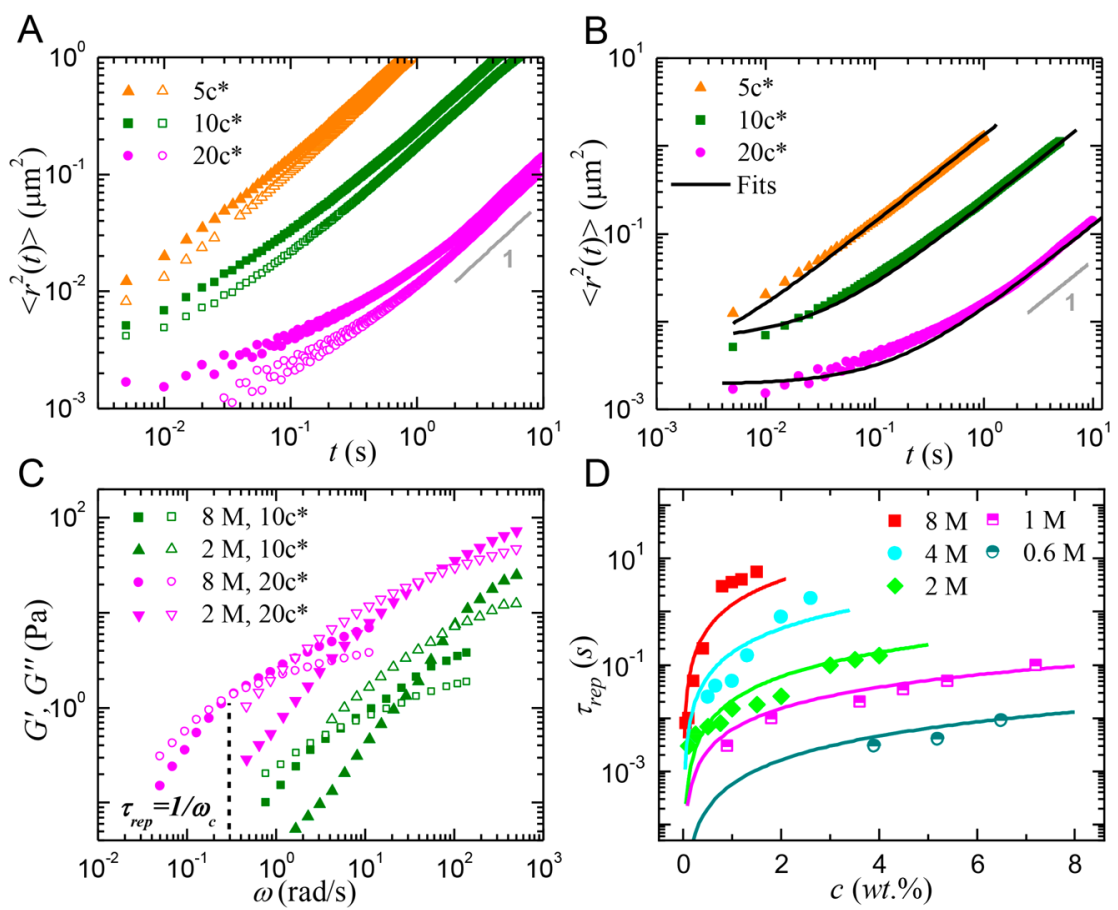

Figure 2. (A) The measured MSDs of $200 \mathrm{~nm} \mathrm{NP}$ in $2 \mathrm{M}$ (empty) and $8 \mathrm{M}$ (solid) PEO solutions. The overlap concentrations are $c^{*}=0.10$ and 0.04 wt \% for 2 and $8 \mathrm{M} \mathrm{PEO}$, respectively. The slope of 1 is drawn to show the linear long-time regime. (B) The fits of measured MSDs by the Maxwell model for $8 \mathrm{M}$ PEO. (C) The storage and loss moduli (MW $\left.=8 \mathrm{M}, c=20 c^{*}\right)$ that were obtained from the rheological measurement offer a way to estimate $\tau_{\text {rep }}=1 / \omega_{\mathcal{c}}$, where $\omega_{\mathrm{c}}$ is the angular frequency at the crossing point of two moduli. (D) The measured $\tau_{\text {rep }}$ as a function of PEO concentrations for $8,4,2,1$, and $0.6 \mathrm{M}$ PEO, respectively. The solid curves are the theoretical predictions based on $\tau_{\text {rep }}=\tau_{0} N^{3} / N_{\mathrm{e}}(1) c^{3(1-v) /(3 v-1)}$ for each MW.

with a size exceeding the adjacent mesh size to diffuse over the confinement cage, which is termed thermally induced activated hopping, has been proposed recently. ${ }^{23,26}$ In particular, the activated hopping of the NP can occur by overcoming a free energy barrier between neighboring cages. Although a few theoretical studies have investigated the possibility of the activated hopping occurring in crowded solutions, the detailed microscopic dynamics and statistical feature of the NP remain elusive. The understanding and characterization of the activated hopping dynamics of NPs in crowded solutions are largely hindered by the absence of reliable experimental data, especially considering the difficulty for common correlation spectroscopy to measure DPD and non-Gaussianity. ${ }^{18,21}$ In addition, experimentally clarifying the factors influencing the activated hopping in crowded solutions is urgently expected by numerical investigations. ${ }^{27-30}$ Note that the mechanism of the activated events has been applied to various systems from glassy material to nanocrystal; ${ }^{31-33}$ we believe that a systematic investigation of the activated hopping would draw broad research interest in nanoscience.

Herein, we conduct experimental investigation to identify the occurrence of the activated hopping and clarify the associated features and time scales with a wide range of concentrations and molecular weights. We will show that the diffusion of NPs in crowded solutions can be described as a superposition of the activated hopping and the slow relaxation 

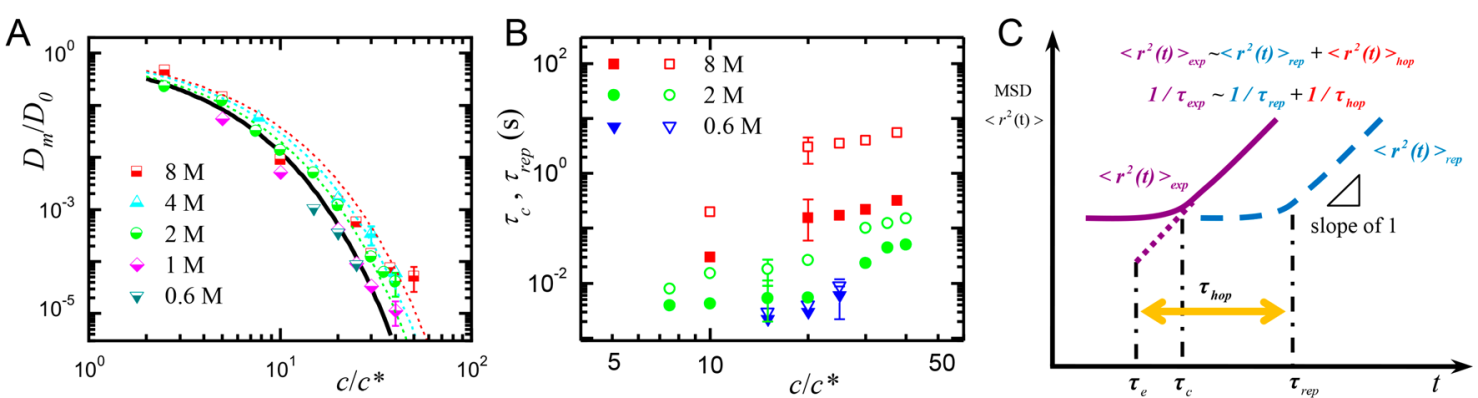

Figure 3. Essential differences of the NP's diffusion measured in crowded solutions compared with previous results in dilute solutions: (A) The enhancement of the measured diffusivity $D_{\mathrm{m}} / D_{0}$. The solid curve is the hydrodynamic model, and the dotted curves fit the tendency of the diffusivity of 2, 4, and $8 \mathrm{M}$ solutions, respectively. Larger values of $D_{\mathrm{m}} / D_{0}$ associated with higher MWs suggest the additional contribution of longdistance hopping in crowded solutions. (B) The earlier transition shown by the comparison between the measured crossover time $\left(\tau_{\mathfrak{c}}\right.$, solid symbols $)$ and the corresponding reptation time $\left(\tau_{\text {rep }}\right.$, empty symbols $)$. Smaller $\tau_{\mathrm{c}}$ indicates the earlier transition. The error bars are estimated on the basis of the measurement uncertainties. (C) A schematic illustration about the influence of NP's activated hopping motion on the MSD, leading to an earlier transition and an enhanced diffusivity as observed in our experiments.

of the polymer solution. We will reveal the distinct timedependent non-Gaussian stochastic behavior of the activated hopping in crowded solutions, which is different from regular hopping diffusion in dilute solutions. We further propose a scaling law to recognize the dimensionless activated hopping time scales, which might be helpful to estimate the occurrence of the activated hopping in different systems.

\section{RESULTS AND DISCUSSION}

In our experiment, we measured the motions of fluorescent polystyrene NPs (Thermo-Fisher) in poly(ethylene oxide) (PEO, Sigma) aqueous solutions by particle tracking microscopy (Figure 1A). We conducted statistical analysis based on the particle tracking results to quantify nonGaussianity based on DPD, which is hardly provided by other techniques including dynamic light scattering. The particle tracking (see the Experimental Methods for more details) was performed as illustrated in our previous work. ${ }^{21}$ The experiments were carried out by varying NP size (diameter $d$ from 100 to $500 \mathrm{~nm}$ ), PEO molecular weight (MW from 0.6 to $8 \mathrm{M} \mathrm{Da}$ ), and PEO concentration ( $c$ from $5 c^{*}$ to $40 c^{*}$, where $c^{*}$ is the overlap concentration $\left.{ }^{34}\right)$. PEO molecules tend to entangle with each other when the concentration $c$ exceeds the entanglement concentration $c_{\mathrm{e}}=$ $N_{\mathrm{e}}(1)^{0.75} c^{*} \sim 7 c^{*}\left(N_{\mathrm{e}}\right.$ is the number of Kuhn monomers in an entanglement strand, and $N_{\mathrm{e}}(1) \approx 14$ in the melt). ${ }^{2,34}$ The positions of the NPs in the image were determined with a subpixel precision (160 nm/pixel for Andor 897 EMCCD), with a resolution of the measured MSD reaching approximately $0.002 \mu \mathrm{m}^{2}$.

As shown in Figure 1A, in the highly crowded solutions used in the present experiments, unusual long-distance motion of the NP still exists, which occurs between the "rattling" motions of the NP (more examples of such long displacements of different NPs are shown in Figure S3). The long displacements are considered to be the direct signals of the hopping behavior of the NPs. ${ }^{11,20,21,35}$ The trajectory implies that the NP moves in a waiting-hopping manner; i.e., the NP needs time to hop as it is strongly confined by the crowded polymer networks. We measure the temporal variation of the displacements (Figure 1B) to highlight the intermittent long-distance hops. In contrast to the dilute solutions, the diameter of the NP is usually larger than the typical entanglement length scale, termed tube diameter $d_{\mathrm{t}}$, in the present PEO solutions. Thus, we focus on the hopping behavior of the "large" NPs subjected to the constraints in the entangled polymer solutions.

From the NP's trajectories, the ensemble-averaged MSD can be calculated. Figure $2 \mathrm{~A}$ presents the measured MSDs of 200 $\mathrm{nm} \mathrm{NP}$ in 2 and $8 \mathrm{M}$ PEO solutions $\left(c=5-20 c^{*}\right)$ as examples. Each curve experiences a transition from the short-time sublinear regime to the long-time linear regime, similar to what exists in dilute solutions. ${ }^{21}$ The NP's MSDs are subdiffusive at short times as the NP is confined by the crowding. After a crossover time $\left(\tau_{\mathrm{c}}\right)$, the MSD recovers to the long-time Fickian behavior. Like the "anomalous yet Fickian" phenomenon, ${ }^{19,20}$ the long-distance hops result in anomalous behavior that is characterized by the DPD and the nonGaussian parameter as we will analyze later. To determine the crossover time, here we introduce the Maxwell model of the non-Newtonian fluid to fit the experimental data of the MSDs with a single crossover time, ${ }^{36,37}$

$$
\left\langle r^{2}(t)\right\rangle=\frac{k_{\mathrm{B}} T}{\pi d G_{\mathrm{e}}}\left(t / \tau_{\mathrm{c}}+1\right)
$$

where $k_{\mathrm{B}}$ is the Boltzmann constant, $T=298 \mathrm{~K}$ is the room temperature, and $G_{\mathrm{e}}$ is the plateau modulus measured from bulk rheology. The measured MSDs are consistent well with the fit curves (Figure 2B), by which we can accurately obtain the crossover time $\tau_{\mathrm{c}}$ (the data of measured $\tau_{\mathrm{c}}$ can be found in Section S2).

In addition, reptation time $\tau_{\text {rep }}$ is known to be the typical relaxation time scale after which the diffusion exhibits a longtime linear behavior. ${ }^{38,39}$ The bulk rheology measurement obtaining the storage and loss moduli offers a way to measure the reptation time $\tau_{\text {rep }}=1 / \omega_{\mathcal{c}}$ where $\omega_{\mathrm{c}}$ is the angular frequency at the crossing point of two moduli (Figure 2C). The measured $\tau_{\text {rep }}$ for various $\mathrm{MW}$ and $c$ are presented in Figure $2 \mathrm{D}$ to show good agreement with the theoretical prediction $\tau_{\text {rep }}=\tau_{0}\left(N^{3} / N_{\mathrm{e}}(1)\right) c^{3(1-\nu) /(3 \nu-1)}\left(N=\mathrm{MW} / \mathrm{M}_{0}\right.$, the number of Kuhn monomers per chain; $M_{0}=140$, the Kuhn monomer molar mass of $\mathrm{PEO} ; \nu=0.588$, the Flory exponent). ${ }^{2,34}$

However, we notice two essential differences in our measured results shown in Figure 2 compared with previous results in dilute solutions. First, we observe an enhanced diffusivity of NPs due to the long-distance hops. Figure 3A shows the variation of the normalized diffusivity $D_{\mathrm{m}} / D_{0}\left(D_{0}\right.$ is the diffusivity in pure solvent) against the normalized 

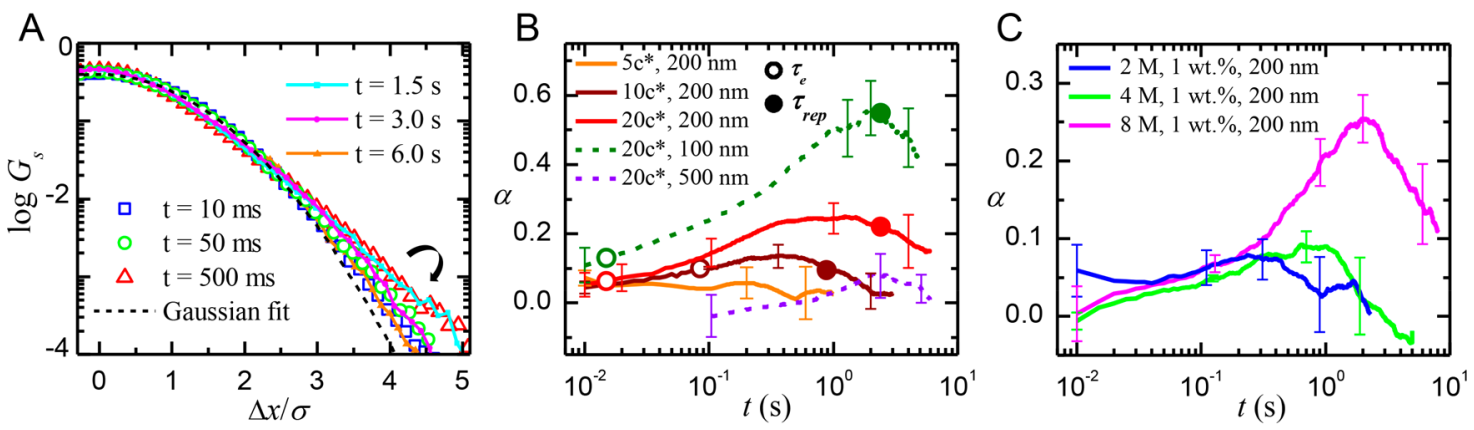

Figure 4. Non-Gaussian statistical behaviors of hopping. (A) The normalized DPD (positive half) vs $\Delta x / \sigma$ of a $200 \mathrm{~nm} \mathrm{NP}$ in an $8 \mathrm{M}$ PEO solution $\left(c=20 c^{*}\right)$ from $t=10 \mathrm{~ms}$ to $t=6 \mathrm{~s}$. The dashed curve is the standard Gaussian distribution. (B) The comparison of the temporal variation of non-Gaussian parameter $\alpha$ in $8 \mathrm{M}$ PEO solutions. Open circles and closed circles are plotted to show the relaxation time $\tau_{\mathrm{e}}\left(\tau_{\mathrm{e}}=\tau_{0} N_{\mathrm{e}}{ }^{2} \sim c^{-2.64}\right)$ and the reptation time $\tau_{\text {rep }}\left[\tau_{\text {rep }}=\tau_{0}\left(N^{3} / N_{\mathrm{e}}(1)\right) c^{3(1-\nu) /(3 \nu-1)} \sim c^{1.5} \mathrm{MW}^{3}\right]$, respectively. (C) The influence of MW on $\alpha$. The error bars are based on the standard deviations of the measured data. Here, (B) and (C) demonstrate that the beginning time of the descent stage of the non-Gaussianity is positively correlated to both MW and $c$, while the onset time of the ascent stage is only sensitive to $c$.

concentration $c / c^{*}$, which clearly shows the enhanced diffusion of NPs when the molecular weight is sufficiently large $(\mathrm{MW} \geq$ $2 \mathrm{M})$. We observe that the experimental data when $c / c^{*}>10$ shift upward from the commonly used hydrodynamic model for dilute solutions ${ }^{38,40}$ (solid curve in Figure $3 \mathrm{~A}: \mathrm{D}_{\mathrm{m}} / \mathrm{D}_{0}=$ $\left.\exp \left[-0.8\left(c / c^{*}\right)^{0.76}\right]\right)$. As shown by the colorful dotted curves, the enhancement of the diffusivity when $M W \geq 2 \mathrm{M}$ is observed to be MW dependent. We stress that this enhanced diffusivity due to the long-distance hops in crowded solutions cannot be explained by the regular hopping in dilute solutions, because the network mesh of the confinement cage is smaller than the NP. In crowded solutions, the NP's diffusive motion was usually considered to be affected by the flow of the surrounding polymer liquids described by the hydrodynamic model. $^{21,22,37,38}$ However, Figure $3 \mathrm{~A}$ clearly indicates a new mechanism causing the long-distance hops and the MWdependent enhancement of the diffusivity beyond the prediction of the existing model. The contribution of this mechanism becomes noticeable when $\mathrm{MW} \geq 2 \mathrm{M}$, suggesting the effect of a critical value of MW.

Second, as a relaxation time characterizing the transition from the subdiffusive regime to the long-time linear regime, the reptation time $\tau_{\text {rep }}$ is supposed to be equivalent to $\tau_{\mathcal{c}}$ which has been verified in dilute solutions. ${ }^{21,37}$ However, Figure $3 \mathrm{~B}$ shows that in crowded solutions as used in the present experiment the crossover time $\tau_{\mathrm{c}}$ obtained from MSD becomes unexpectedly smaller than $\tau_{\text {rep }}$ once MW exceeds $2 \mathrm{M}\left(\tau_{\text {rep }}\right.$ and $\tau_{\mathrm{c}}$ are measured, respectively, as shown in Figure 2, and their values are provided in Section S2). For $8 \mathrm{M} \mathrm{PEO}, \tau_{\mathrm{c}}$ is about 1 order of magnitude smaller than $\tau_{\text {rep. }}$. This MW-dependent earlier transition indicated by crossover time $\tau_{\mathcal{c}}$ combined with the aforementioned MW-dependent enhanced diffusivity, depicts an extra contribution of the long-distance hops on MSD compared with the reptation model as schematically demonstrated by Figure 3C. The experimentally measured MSD curve $\left\langle r^{2}\right\rangle_{\exp }$ (purple solid curve) appears to shift upward from the reptation prediction $\left\langle r^{2}\right\rangle_{\text {rep }}$ (blue dashed curve).

Except for the above anomalies, we further employ DPD to identify the statistical characteristic of the long-distance hopping motion in crowded polymer solutions (Figure 4A). The larger hopping displacements, corresponding to the larger $\Delta x / \sigma$ in Figure $4 \mathrm{~A}$, result in the non-Gaussian "fat tailed" DPD. Therefore, we are able to evaluate the occurrence of the hopping behavior based on the "fat tailed" DPD. According to the probability density function of a standard Gaussian distribution of a variable $y: G(y)=\frac{1}{\sqrt{2 \pi}} \exp \left[-y^{2} / 2\right]$, we substitute $y=\Delta x / \sigma$ into it to establish the standard Gaussian distribution of the normalized displacement $G_{s}=\frac{1}{\sqrt{2 \pi}} \exp \left[-(\Delta x / \sigma)^{2} / 2\right]$. Compared with the standard Gaussian distribution, the deviation at the fat tail $(\Delta x / \sigma>3$ in Figure 4A) represents a considerable increase of the occurrence of the long-distance hop. This tail distribution decays linearly on the logarithmic plot (implying $G_{\mathrm{s}} \sim$ $\exp [-\Delta x / \sigma])$ with a slope varying from -1.9 to -2.6 , showing an intriguing behavior similar to the "diffusing diffusivity". ${ }^{41}$ Furthermore, we observe a clear two-stage variation of the tailed distribution. As shown in Figure 4A for $200 \mathrm{~nm}$ NPs in $8 \mathrm{M}$ PEO $\left(c=20 c^{*}\right)$, the fat tail becomes more obvious when $t$ exceeds $\sim 0.1 \mathrm{~s}$, whereas it declines gradually when $t$ is approximately $>1.5 \mathrm{~s}$ so that the DPD eventually returns to be Gaussian.

It is a common practice using the non-Gaussian parameter, $\alpha=\left(\left\langle\Delta x^{4}\right\rangle /\left(3\left\langle\Delta x^{2}\right\rangle^{2}\right)\right)-1$, to quantitatively describe the time-varied non-Gaussian trend. ${ }^{5,18}$ We will show below that the variation of $\alpha$ in the present experiment not only depends on NP size and PEO MW and concentration but also manifests a distinct behavior compared with the regular hopping diffusion. Generally, $\alpha=0$ represents a Gaussian process, for instance, a simple Brownian motion. The positive values of $\alpha$ in the present study indicate the existence of the non-Gaussian "fat tails" (Figure 4B). The more positive $\alpha$, the more obvious is the fat tail. We observe that the non-Gaussianity usually has a range of $0.1<\alpha<0.3$ in the present experiments, which is significant enough compared with the values of $\alpha=0.05-0.1$ in previous experiments or simulations in polymer solutions. $^{21,42}$ Figure $4 \mathrm{~B}$ shows the influence of PEO concentration and NP size on $\alpha$ at a fixed molecular weight of $8 \mathrm{M}$. At low concentration $c=5 c^{*}$ (orange solid curve), the values of $\alpha$ are from 0 to 0.05 , showing a very weak non-Gaussianity considering the measurement uncertainty $( \pm 0.04$ to \pm 0.1$)$. Therefore, we conclude with confidence that the results with $\alpha$ $\geq 0.1$ are clearly non-Gaussian. As the concentration increases, all curves of $c \geq 10 c^{*}$ exhibit an ascent stage of $\alpha$ at short times, followed by a descent stage at long times. Previous works have shown that in regular hopping diffusion in dilute solutions the non-Gaussianity $\alpha$ maintains a constant value of approximately $0.1 .^{21,42}$ Thus, this ascent-to-descent two-stage variation of $\alpha$ is a distinct statistic characteristic of the long- 
distance hopping in crowded solutions. The three solid curves illustrate that the plateau value of $\alpha$ gets higher with the increasing concentration. At short times $t \sim 10^{-2}-10^{-1} \mathrm{~s}, \alpha$ of $20 c^{*}$ is larger than that of $10 c^{*}$. If we choose an $\alpha$ of approximately 0.1 as the beginning of a clear ascent stage, we observe an earlier onset of the ascent stage with a higher concentration like $c=20 c^{*}$. Contrarily, the beginning of the descent stage of $10 c^{*}(\sim 0.5 \mathrm{~s})$ is earlier than that of $20 c^{*}$ (>1 s). This temporal variation was considered to be an important factor to unveil the anomalous events and to distinguish dominant mechanisms. ${ }^{43} \mathrm{We}$ find that the beginning of the ascent stage and the descent stage are closely related to the local relaxation time $\tau_{\mathrm{e}}\left(\tau_{\mathrm{e}}=\tau_{0} N_{\mathrm{e}}{ }^{2}\right.$, where $\tau_{0}$ is the relaxation time of a monomer) of an entanglement strand ${ }^{34}$ and the longtime relaxation time $\tau_{\text {rep }}$ [reptation time $\tau_{\text {rep }}=\tau_{0}\left(N^{3} / N_{\mathrm{e}}(1)\right)$ $\left.c^{3(1-\nu) /(3 \nu-1)}\right],{ }^{2,34}$ respectively. We mark $\tau_{\mathrm{e}}$ and $\tau_{\text {rep }}$ in Figure 4B with open and closed circles, respectively, to show that they are indeed good indicators of the ascent and descent stages. The mechanism of why $\tau_{\mathrm{e}}$ and $\tau_{\text {rep }}$ are involved will be discussed later. It is worth noting that $\tau_{\mathrm{e}}$ monotonically decreases with the concentration $\left(\tau_{\mathrm{e}} \sim c^{-2.64}\right)$ but $\tau_{\text {rep }}$ monotonically increases with $c$ and $\mathrm{MW}\left(\tau_{\text {rep }} \sim c^{1.5} \mathrm{MW}^{3}\right)$, which is also in accordance with our observation. In addition, from the two dashed curves in Figure 4B, we can tell that smaller NPs are more likely to hop and thus cause larger non-Gaussianity. For instance, the non-Gaussian parameter $\alpha$ of $100 \mathrm{~nm}$ NP (green dashed curve) is up to 0.5 , indicating that the non-Gaussianity of the hopping diffusion is significantly larger than the predicted values in previous theoretical works. ${ }^{28,30}$

Figure 4C presents the results of different molecular weights at a fixed concentration of $1 \mathrm{wt} \%$. The ascent-to-descent twostage variation also emerges, with $\alpha$ increasing with the molecular weight. According to Figure 4B,C combined, the long-distance hopping events in our experiments, as characterized by the relatively high non-Gaussianity, mainly occur at intermediate times. All curves of Figure $4 \mathrm{C}$ in the ascent stage display a similar trend, suggesting the weak influence of MW on the onset time of the ascent stage, although the decay of the non-Gaussianity starts later for a larger MW. Therefore, we can conclude that the beginning time of the descent stage of the non-Gaussianity is positively correlated to both molecular weight MW and concentration $c$, while the onset time of the ascent stage is only sensitive to the concentration. This result therefore suggests the influences of the entanglement time $\tau_{\mathrm{e}}$ and the reptation time $\tau_{\text {rep }}$ on the beginning times of the ascent stage and the descent stage, respectively, as $\tau_{\text {rep }}$ is dependent on both MW and $c$ while $\tau_{\mathrm{e}}$ is not influenced by MW.

The above experiments have provided results in three aspects to characterize the long-distance hopping of large NPs $\left(d>d_{t}\right)$ in entangled polymer solutions. First, we observe intermittent long-distance hops occurring between the "rattling" motions of the NPs. Second, we find an enhanced diffusion (Figure 3A,B), which is attributed to the long hopping displacements. The enhancement of the MSD simultaneously causes an upward shift and an earlier transition of the measured MSD, as shown in Figure 3C. This shift demonstrates an extra contribution beyond the prediction of the well-established reptation model. ${ }^{26}$ Third, we reveal an ascent-to-descent two-stage variation of the non-Gaussianity $\alpha$ at intermediate times. This statistic feature in crowded solutions is distinct from the behavior of the regular hopping in dilute solutions. Among the above results, the shift of the MSD and the ascent-to-descent non-Gaussianity appear to be different from the regular hopping in dilute solutions. Taken together, these results suggest a new mechanism of the longdistance motion under the confinement of the crowded environment.

We argue that the thermally induced activated hopping is responsible for the long displacement observed in the experiments. ${ }^{23,26,44}$ To diffuse in such crowded solutions, the NPs can either intermittently hop between confinement network cages or passively move with the surrounding polymer liquids. As the network mesh size is usually much smaller than the NP's diameter in crowded polymer solutions, the most possible mechanism for NPs to hop is by thermally induced activated hopping rather than regular hopping. The microscopic process of the activated hopping can be understood as the polymer chains slip around the NP and adjust the structure to open a loop between two neighboring confinement cages. ${ }^{23}$ The NP needs to overcome an energy barrier in this process to intermittently hop through the open loop, which is mainly due to elastic deformation of the network strands during the activated event. $^{26}$ The two-stage time-dependent nonGaussianity excludes the activated hopping either at short times or at long times, which could be explained from the perspective of time scale. First, the NPs have to wait for a proper time to trigger an activated hop. The microscopic restraint from the adjacent network cage can be characterized in terms of the local relaxation time $\tau_{\mathrm{e}}$ of the confinement chains. We can only detect the activated event of NP breaking through the confinement cage when $t>\tau_{\mathrm{e}}$, associated with the ascent stage of the non-Gaussianity. Second, the descent stage is a result of the long-time restoration to Gaussianity. Such restoration corresponds to the slow relaxation of the polymer chains with a reptation time scale $\tau_{\text {rep. }}$. The competition between the activated hopping and the slow relaxation governs the long-time dynamics when the non-Gaussianity starts to decay. According to the observations in Figure 4B,C, the onset time of the ascent stage is approximately the entanglement time $\tau_{\mathrm{e}}\left(\tau_{\mathrm{e}} \sim c^{-2.64}\right)$ while the beginning time of the descent stage is the reptation time $\tau_{\text {rep }}\left(\tau_{\text {rep }} \sim c^{1.5} \mathrm{MW}^{3}\right)$. Thus, the clear non-Gaussianity caused by the activated hopping emerges when $\tau_{\mathrm{e}}<t<\tau_{\text {rep }}$.

It is then natural to ask how to estimate the time scale of such activated hopping. On the basis of the above analysis, we emphasize that the NP's motion when $t>\tau_{\mathrm{e}}$ can be considered as a superposition of the activated hopping and the long-time reptation. As illustrated by Figure 3C, the activated hopping causes a shift of the MSD curve from the reptation prediction $\left\langle r^{2}\right\rangle_{\text {rep }}$ (blue dashed curve) to the experimentally measured value $\left\langle r^{2}\right\rangle_{\text {exp }}$ (purple solid curve). Therefore, we use eq 2 below to estimate the extra contribution $\left\langle r^{2}\right\rangle_{\text {hop }}$ due to the activated hopping by the shift of the MSD: ${ }^{26}$

$$
\left\langle r^{2}(t)\right\rangle_{\exp }=\left\langle r^{2}(t)\right\rangle_{\text {rep }}+\left\langle r^{2}(t)\right\rangle_{\text {hop }}
$$

This relation is extended from Cai et al.'s theory ${ }^{26}$ and has just been applied to entangled polymer solutions. ${ }^{45}$ On the basis of the model of Maxwell fluids, we can approximately define the linear MSD scaling at long times on the basis of the reptation time: $\left\langle r^{2}(t)\right\rangle_{\text {rep }} \approx \frac{k_{\mathrm{B}} T}{\pi d G_{\mathrm{e}}} t \tau_{\text {rep }}$. However, due to the activated hopping, the experimentally measured MSD manifests an earlier transition $\left(\tau_{c}\right)$ and deviates from the above prediction: $\left\langle r^{2}(t)\right\rangle_{\exp } \approx \frac{k_{\mathrm{B}} T}{\pi d G_{\mathrm{e}}} t \tau_{c_{\mathrm{c}}}$. As a result, using $\tau_{\mathcal{c}}, \tau_{\text {rep }}$, and $\tau_{\text {hop }}$ as the 
typical time scales of each MSD term, respectively, eq 2 can be simplified to

$$
1 / \tau_{\mathrm{c}} \approx 1 / \tau_{\text {rep }}+1 / \tau_{\text {hop }}
$$

With $\tau_{\mathrm{c}}$ and $\tau_{\text {rep }}$ obtained in the experiments (Figure 3B), the value of $\tau_{\text {hop }}$ can be evaluated (the data of $\tau_{\text {hop }}$ are provided in Section S3). Our approach can be applied to a wide range of solutions, for instance, $1-8 \mathrm{M} \mathrm{PEO}$ with concentration $c / c^{*}=$ 10-40. The concept of this approach based on the Maxwell model and the relaxation time scales might be extended to other complex fluids.

On the basis of the calculated values of $\tau_{\text {hop }}$, we now propose a scaling law to link the dimensionless time $\tau_{\text {hop }} / \tau_{\mathrm{e}}$ and the dimensionless size $d / d_{\mathrm{t}}$ :

$$
\tau_{\text {hop }} / \tau_{\mathrm{e}}=\lambda \exp \left(d / d_{\mathrm{t}}\right)
$$

The parameter $\lambda$ is about 1.3 by fitting (here, the root mean squared error (RMSE) is less than 1 , and the $R$-square coefficient is approximately 0.8 ). In Figure 5, the measured

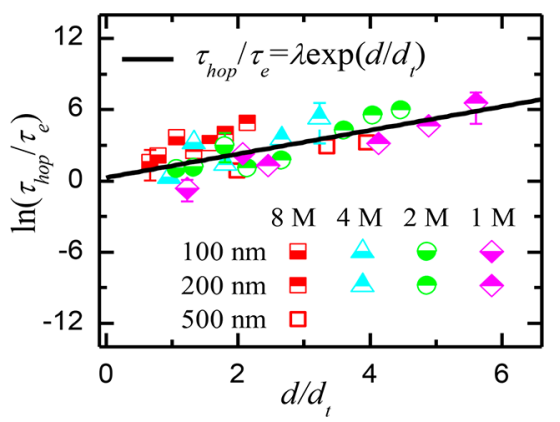

Figure 5. Scaling law of $\ln \left(\tau_{\text {hop }} / \tau_{\mathrm{e}}\right)$ vs $d / d_{\mathrm{t}}$ for different NPs in various PEO solutions. A solid line with a slope of unity is given to show the exponential equation $\tau_{\text {hop }} / \tau_{\mathrm{e}}=\lambda \exp \left(d / d_{\mathrm{t}}\right)$.

data display good agreement with the tendency of the solid line from this scaling law in the range of $1<d / d_{\mathrm{t}}<6$. In contrast to the narrow range around $d / d_{\mathrm{t}} \approx 2$ mentioned in previous works, ${ }^{45}$ our results reveal the characteristics of the activated hopping in a much wider range. Equation 4 is also in line with the theory of Cai et al., ${ }^{26}$ which demonstrates the mechanism that NPs can overcome the strong confinement of the entangled networks by thermally induced activated hopping. Equation 4 can be elucidated by the concept of energy landscape. The waiting-hopping behavior shown in Figure 1A indicates the NPs hop between entangled network cages. The energy barrier $\Delta G$ for the activated hopping can be described on the basis of the Boltzmann description of the activated phenomenon, ${ }^{32}$ thus yielding the hopping frequency $f_{\text {hop }}=1 /$ $\tau_{\text {hop }} \sim \exp \left(-\Delta G / k_{\mathrm{B}} T\right)$, consistent with eq 4 . This exponential relation of the hopping frequency is also found to be similar to the Arrhenius relation, which might be used to phenomenologically describe how the activated events are related to the energy barrier in different systems. ${ }^{35}$ We notice that the prefactor $\lambda=1.3$ in eq 4 is usually 1 order of magnitude smaller than the energy barrier term $\exp \left(d / d_{\mathrm{t}}\right)$, suggesting that the activated hopping is mainly influenced by the energy barrier rather than the prefactor.

The energy barrier can be estimated by considering the elastic deformation energy due to slipping or stretching of some strands around the NP, though theoretical consensus has not been reached yet. One might estimate the deformation energy by including all the network strands affected by the NP in its occupied volume, which is thus assumed to follow $d^{3} /$ $d_{t}{ }^{3}$. ${ }^{44}$ While a theoretical model of Rubinstein's group proposed linear dependence of the energy barrier on dimensionless size $d / d_{\mathrm{t}}{ }^{23,26}$ Analogous to the Boltzmann relation $1 / \tau_{\text {hop }} \sim \exp \left(-\Delta G / k_{\mathrm{B}} T\right)$, the scaling of eq $4\left[\tau_{\text {hop }} \sim\right.$ $\left.\exp \left(d / d_{\mathrm{t}}\right)\right]$ suggests that the energy barrier is linearly proportional to the dimensionless NP size, i.e., $\Delta G \sim$ $k_{\mathrm{B}} T\left(d / d_{\mathrm{t}}\right)$, rather than varying with the NP volume $\left(\sim d^{3}\right)$. The linear dependence of $\Delta G \sim k_{\mathrm{B}} T\left(d / d_{\mathrm{t}}\right)$ here implies that it is not necessary for all strands to deform in the same way during a single activated hopping event. Note that for small barriers Dell and Schweizer showed that the dependence on $d /$ $d_{\mathrm{t}}$ was of a power law form with an exponent much less than $3{ }^{44}$ Therefore, for "large" NPs $\left(d / d_{t}>1\right)$ in entangled solutions, the linear dependence results in a lower energy barrier compared with a cubic relation based on the NP's volume. This result explains that the energy barrier of smaller NPs is much lower so that smaller NPs can hop more easily
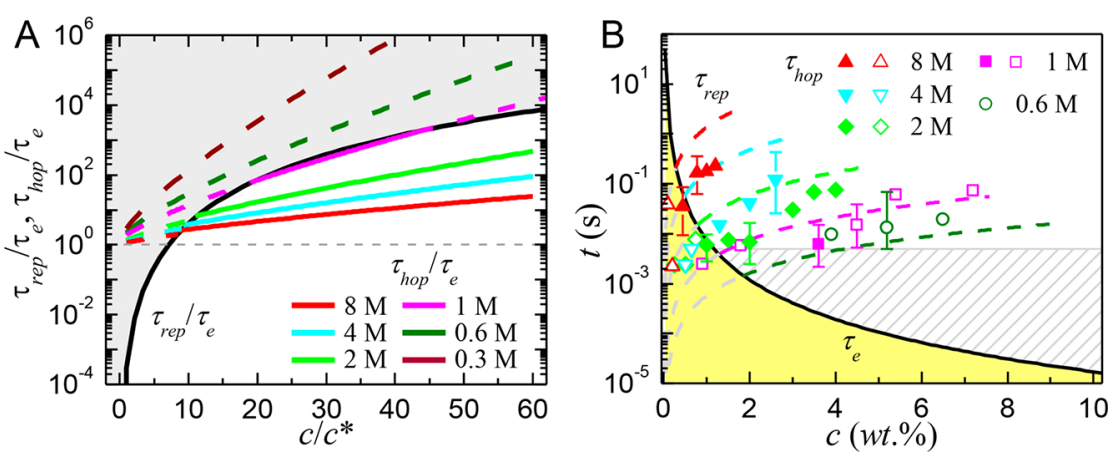

Figure 6. Competition between hopping dynamics and polymer relaxations based on their time scales. (A) A comparison between $\tau_{\text {hop }} / \tau_{\mathrm{e}}$ (colorful curves) and $\tau_{\text {rep }} / \tau_{\mathrm{e}}$ (black solid curve), showing the competition between hopping dynamics and polymer relaxations. The activated hopping occurs in the solid section of each colorful curve in the bright region. The dashed horizontal line represents a threshold $\tau_{\text {hop }}=\tau_{\mathrm{e}}$ below which activated hopping cannot occur. (B) A phase diagram showing the comparison among $\tau_{\text {hop }}$ (different symbols), $\tau_{\mathrm{e}}$ (black solid curve), and $\tau_{\text {rep }}$ (dashed curves with different colors), by which different regions are obtained to characterize the NP dynamics. Different colors represent different molecular weights: $8 \mathrm{M}$ (red), $4 \mathrm{M}$ (cyan), $2 \mathrm{M}$ (green), $1 \mathrm{M}$ (purple), and $0.6 \mathrm{M}$ (olive). The hopping behavior exhibiting the two-stage non-Gaussianity is only observed when $\tau_{\mathrm{e}}<\tau_{\text {hop }}<\tau_{\text {rep }}$, as displayed by the solid symbols. Empty symbols represent the cases in which the hopping motion was not detected. The yellow region means $t<\tau_{\mathrm{e}}$ where hopping cannot occur as NPs are still constrained by the entanglements. The gray horizontal line represents the time resolution $t=5 \mathrm{~ms}$ in the present experiment. 
than larger ones, as shown in Figure 4B. The measured data become more scattered when $d / d_{\mathrm{t}}$ is approximately $<2$, because the above explanation based on large NPs $\left(d>d_{\mathrm{t}}\right)$ becomes invalid.

Also note that the exponential scaling law of the thermally activated hopping exists in many other systems like nanocrystals, glassy materials, and hydrogels; ${ }^{31,32,35,46,47}$ our finding is helpful to better understand the activated hopping mechanism in crowded systems. For the practical application of nanomedicine, the temperature effect has been shown to improve drug delivery. ${ }^{48,49}$ In the regular diffusion, an increase in temperature will decrease the viscosity of medium and thus enhance the diffusion. In crowded solutions, an increase in temperature could work differently from a synergetic effect, combining the activated hopping and the decreased viscosity. According to the Boltzmann description of the activated frequency, i.e., $f_{\text {hop }}=1 / \tau_{\text {hop }} \sim \exp \left(-\Delta G / k_{\mathrm{B}} T\right)$, an increase in temperature can trigger more activated events, which might provide a new mechanism to enhance the delivery of nanomedicines in crowded biological microenvironments.

We then provide Figure $6 \mathrm{~A}$ based on the dimensionless results to elucidate when the activated hopping occurs. The criterion of the hopping-dominant region, i.e., $\tau_{\mathrm{e}}<\tau_{\text {hop }}<\tau_{\text {rep }}$, excludes the possibility of hopping in the dark region above the black solid curve and the region below the gray dashed line. For instance, for the dashed curves of $0.3-0.6 \mathrm{M}$ solutions, $\tau_{\text {hop }}$ is even larger than $\tau_{\text {rep, }}$ which means the solutions have been dominated by reptation before any activated hopping occurs. The activated hopping with non-Gaussian DPD can only occur in the solid section of each colorful line in the bright region. This is in good agreement with our observation of the nonGaussian hopping behaviors in 2-8 $\mathrm{M}$ solutions when concentration $c \geq 10 c^{*}$ (Figure 3). Figure 6A illustrates that higher MW facilitates the occurrence of the activated hopping as observed in our experiments, in accordance with previous theoretical predictions. ${ }^{26,28,45}$ The onset cutoff concentration around $c / c^{*} \sim 10$ will increase with the deceasing MW. Note that for the $1 \mathrm{M}$ solution there is a termination cutoff concentration at about $c / c^{*}=43$, beyond which the hopping diffusion will hardly occur as the reptation becomes dominant. We underscore that increasing $c$ or MW or decreasing $d$ will enhance the contribution of the activated hopping. This result offers more means to generate long-distance motion in a crowded environment rather than an increase in temperature only.

We summarize our experimental data of $\tau_{\text {hop }}$ in Figure $6 \mathrm{~B}$ based on the framework of Figure 6A. In Figure 6B, the cases in which clear hopping motion results in two-stage nonGaussianity are marked by solid symbols. The solid curve is $\tau_{\mathrm{e}}$ which is independent of the molecular weight, and the dashed curves represent the experimentally defined values of $\tau_{\text {rep }}$ in various PEO solutions. The yellow region means $t<\tau_{\mathrm{e}}$, where activated hopping cannot occur as NPs are still constrained by the entanglements. For 2-8 M solutions, the activated hopping behavior with non-Gaussian fat-tailed DPD occurs when $\tau_{\mathrm{e}}<$ $\tau_{\text {hop }}<\tau_{\text {rep }}$, which means the solid symbols should locate between the solid curve $\left(t=\tau_{\mathrm{e}}\right)$ and the corresponding dashed curve $\left(t=\tau_{\text {rep }}\right)$ with the same color. It is noted that two solid symbols (a red triangle for $8 \mathrm{M}$ at $c=0.4 \%$ and a green diamond for $2 \mathrm{M}$ at $c=1 \%$ ) are not strictly located on the right side of the solid curve. Considering the measurement uncertainty as marked by the error bars, however, we believe that they actually satisfy the statement above. For $1 \mathrm{M}$ solutions, there exists one solid symbol at $c=3.6 \%\left(c=20 c^{*}\right)$, which falls into the range of our prediction that hopping might be detected approximately at $20<c / c^{*}<35$ (the solid part of the purple curve in Figure 6A). For another symbol of $1 \mathrm{M}$ solution at $c=4.5 \%\left(c=25 c^{*}\right)$, we mark it as empty because the measured non-Gaussian parameter $\alpha$ is below 0.05 , and we are unable to discern if activated hopping occurs by the twostage variation of $\alpha$. For $0.6 \mathrm{M}$ solutions, we do not detect noticeable non-Gaussian activated hopping behaviors, as predicted from Figure 6A. The above result sheds light on the enhancement of "rare" long-distance hopping that might play a critical role of biofunctioning in crowded biological systems and drug delivery in nanomedicines. ${ }^{20,25,50-53}$ Real biological systems are more complicated than the PEO solution mentioned here. Nonetheless, many crowded biological systems display characteristics similar to crowded polymer solutions, for example, their macromolecular nature, viscoelasticity, and heterogeneous microscopic structures. Thus, we believe that the activated hopping could occur as well in these biological systems and serve as a new microscopic mechanism to enhance the local transport. The activated hopping and its effect of overcoming strong local confinement might be further strengthened by introducing excitation from external fields like temperature or ultrasound. ${ }^{54,55}$

\section{CONCLUSION}

In summary, we clarify the non-Gaussian stochastic nature and the time-dependent activated hopping dynamics of NPs in crowded polymer solutions. We observe the long-distance hops of NPs that were thought to be unlikely in crowded solutions. These long-distance hops cause an enhancement of the diffusivity and the MSD beyond the prediction of the existing models and an ascent-to-descent two-stage variation of the non-Gaussianity distinct from the statistic behavior of the regular hopping diffusion in dilute solutions. Therefore, we identify the thermally induced activated hopping as a new mechanism and a non-negligible source for NP's diffusive motion in the crowded solutions. The diffusion of NPs in a crowded solution can be deemed as a superposition of the activated hopping and the slow relaxation with the surrounding solutions. The microscopic mechanism of the activated hopping can be understood as the polymer chains slip around the NP to open a loop between two neighboring confinement cages, and the NP hops through the loop by overcoming an energy barrier.

To characterize the activated hopping, we examine the time scale of the activated hopping and demonstrate the activated hopping based on statistical analysis. The ascent-to-descent two-stage non-Gaussianity reveals the competition among the short-time relaxation of the local entanglement cage, the activated hopping dynamics, and the long-time relaxation. We therefore propose an exponential scaling law $\tau_{\text {hop }} / \tau_{\mathrm{e}} \sim \exp (d /$ $d_{t}$ ) to characterize the hopping time scale. The activated hopping motion of large NPs in entangled polymer solutions can be observed only when $\tau_{\mathrm{e}}<\tau_{\text {hop }}<\tau_{\text {rep }}$, which is triggered by overcoming the free energy barrier $\Delta G \sim k_{\mathrm{B}} T\left(d / d_{\mathrm{t}}\right)$ in the thermally activated process. This energy barrier is found to be linearly proportional to the dimensionless NP diameter rather than varying with the NP volume. In addition, the occurrence of the activated hopping is found to depend on the NP size and the polymer MW and concentration. In contrast to the regular hopping in dilute solutions, the thermally induced activated hopping acts as an extra affect to enhance local transport in 
crowded solutions with proper parameters such as smaller NPs or higher MWs. Our approach in the present study based on the basic relaxation times and typical length scale might be applied to other complex systems with mesoscopic structures, and our findings expand the insight into the anomalous activated hopping in crowded environments from biological macromolecular systems to porous media. At present, we use a well-controlled model system to investigate the fundamental mechanism regarding the NP's diffusion. It is truly worthwhile to work with real biological media in the future study.

\section{EXPERIMENTAL METHODS}

Preparation of NP-Dispersed PEO Solution. NPdispersed PEO solutions were prepared by adding both PEO powders (Sigma-Aldrich) and fluorescent polystyrene NPs (Thermo-Fisher) in deionized water (Milli-Q). Then, they were adequately mixed by a shaker with a rate of $\sim 60 / \mathrm{min}$ for 3-10 days. The volume fractions of the NPs are ultralow $\left(10^{-4}-10^{-6} \%\right)$, and the particle-particle interaction was rationally neglected. In the blending process, the containers were wrapped by aluminum foil to minimize the loss of fluorescence and the polymer degradation. The prepared samples were maintained for at least 2 days at room temperature before performing the experiment. In addition, a homemade PDMS cell sealed by two coverslips was fabricated to encapsulate the sample solution to avoid evaporation and creep deformation of the sample solution. This simple device is presented in Figure S1.

Particle Tracking Experiment. A particle tracking experiment was performed using an inverted fluorescence microscope (Olympus IX71) with a $100 \times / 1.40$ oil immersed objective. A sensitive EMCCD (Andor 897, $512 \times 512$ pixels) was used to record the images. Using a $100 \times$ objective, the size of a single pixel in the image was approximately $160 \mathrm{~nm}$. The experiment was performed in the subimage mode to fast record the frequency, in which the image field of view was fixed to be $250 \times 80$ pixels (approximately $40.0 \times 12.8 \mu \mathrm{m}$ ). The fastest record frequency in the current experiments was 200 frames per second, corresponding to about a $5 \mathrm{~ms}$ interval between two consecutive images. With the help of a piezo-transducer (Physik Instrument) mounted under the objective, the focus plane of the objective was located more than $10 \mu \mathrm{m}$ above the solution-glass interface to avoid wall interruption. The raw images of 100 and $40 \mathrm{~nm}$ NPs in the current experiment are shown in Figure S2a,d. The observations were performed at 20-40 different positions. At each position, an image sequence containing 3000-8000 frames was captured. The motion of an individual particle was then identified and tracked from the acquired image sequences with ImageJ software and a housewritten Matlab algorithm. For each case, typical raw data of hundreds of thousand NP positions as a function of time were obtained from hundreds of fluorescent NPs. For most cases, three independent experiments were performed to ensure the reproducibility of the measurements. Details of the measurement uncertainty can be found in Section S4.

Image Process. The image process contained three procedures: image filtering, particle identification, and particle tracking. First, image filtering was used to reduce the image noise. Methods like "Gaussian blur" and "contrast sharpen" were used to enhance the spot of the NP in the images (Figure $\mathrm{S} 2 \mathrm{~b}, \mathrm{e})$. Filtering using proper threshold gray scale value was sufficient to remove the noise. Figure $S 2 \mathrm{c}$,f shows very good contrast that enabled us to precisely determine the position of the NP center. After that, for the particle identification, the program first calculated the contour map of the gray scale value and found out the peak region of the particle center position. Then, a fit of the gray scale values in this area based on Gaussian distribution was recorded as the particle center. Theoretically, this method could reach a subpixel precision of determining a NP's horizontal center position. After identifying NPs in each image, the program started tracking NPs between successive frames. In this procedure, an area of about $10 \times 10$ pixels around each particle center was picked out as a search region. The NP appearing in the same search region in the next frame was considered as the same NP. This method works well when the NP concentration is diluted such as in our case, and if there were more than one NP appearing in the same search region, the tracking process would be terminated to avoid the influence of particle collision and particle-particle interaction. The NP displacement can be calculated from the position variation between two consecutive images with a precision about $30 \mathrm{~nm}$.

Bulk Rheological Measurement. Bulk rheological measurements of PEO solutions were performed using a rheometer (Physica MCR301, Anton Parr GmBH) with a cone-plate geometry $(50 \mathrm{~mm}, 0.3 \mathrm{rad})$. The viscosities $\eta$ were measured at the shear rate of $0.01-3000 \mathrm{~s}^{-1}$ (Figure S3). The storage $G^{\prime}$ and loss $G^{\prime \prime}$ moduli as a function of angular frequency $(\omega)$ were measured in dynamic oscillatory experiments, by which we obtained the reptation time $\tau_{\text {rep }}$ as shown in Figure $3 \mathrm{~b}$. These oscillatory measurements were conducted in the predetected linear viscoelastic regime with a constant strain of $4 \%$. All the measurements were conducted at $25{ }^{\circ} \mathrm{C}$ maintained by a Peltier stage.

\section{ASSOCIATED CONTENT}

\section{Supporting Information}

The Supporting Information is available free of charge at https://pubs.acs.org/doi/10.1021/acs.nanolett.0c01058.

S1: Materials and methods, scheme of the experimental cell, process of image filtering and particle identification, typical trajectories of the intermittent long-distance hops, measured viscosity as a function of shear rate, measured storage $G^{\prime}$ and loss $G^{\prime \prime}$ moduli, and calculated values of tube diameters and average mesh sizes; S2: determination of reptation time $\tau_{\text {rep }}$ and plateau modulus $G_{e}$, typical values of $\tau_{c}$ and $\tau_{\text {rep }}$, and experimentally obtained plateau moduli $G_{\mathrm{e}}$ as a function of normalized concentrations; S3: calculation of the hopping time $\tau_{\text {hop }}$, calculated values of $\tau_{\text {hop }}$ of $200 \mathrm{~nm} \mathrm{NP}$ for various PEO, and several measured values of $\Delta t_{\mathrm{hop}}$; 4 : estimation of the measurement uncertainty, three independent measurements of MSD, additional plots for Figure 5, and estimation of the relative uncertainties (PDF)

\section{AUTHOR INFORMATION}

\section{Corresponding Authors}

Guoqing Hu - Department of Engineering Mechanics \& State Key Laboratory of Fluid Power and Mechatronic Systems, Zhejiang University, Hangzhou, Zhejiang 310027, China; ○ orcid.org/0000-0001-9451-5336; Email: ghu@zju.edu.cn

Xu Zheng - State Key Laboratory of Nonlinear Mechanics, Institute of Mechanics, Chinese Academy of Sciences, Beijing 100190, China; 이이.org/0000-0002-2398-9283; Email: zhengxu@lnm.imech.ac.cn 


\section{Authors}

Chundong Xue - State Key Laboratory of Nonlinear Mechanics, Institute of Mechanics, Chinese Academy of Sciences, Beijing 100190, China; School of Optoelectronic Engineering and Instrumentation Science, Dalian University of Technology, Dalian, Liaoning 116024, China; University of Chinese Academy of Science, Beijing 100149, China; ㅈorcid.org/ 0000-0002-5259-4491

Xinghua Shi - National Center for Nanoscience and Technology of China, Beijing 100190, China; University of Chinese Academy of Science, Beijing 100149, China; 이이.org/ 0000-0001-5012-3453

Yu Tian - State Key Laboratory of Tribology, Tsinghua University, Beijing 100084, China; (1) orcid.org/0000-00017742-5611

Complete contact information is available at: https://pubs.acs.org/10.1021/acs.nanolett.0c01058

\section{Notes}

The authors declare no competing financial interest.

\section{ACKNOWLEDGMENTS}

The authors acknowledge the Natural Science Foundation of China (Grant Nos. 11832017, 11572335, 11572334, and 11802054), the Chinese Academy of Sciences Key Research Program of Frontier Sciences (QYZDB-SSW-JSC036), the Chinese Academy of Sciences Strategic Priority Research Program (XDB22040403), and the China Postdoctoral Science Foundation (2019M651106).

\section{REFERENCES}

(1) Babaye Khorasani, F.; Poling-Skutvik, R.; Krishnamoorti, R.; Conrad, J. C. Mobility of Nanoparticles in Semidilute Polyelectrolyte Solutions. Macromolecules 2014, 47 (15), 5328-5333.

(2) de Kort, D. W.; Rombouts, W. H.; Hoeben, F. J. M.; Janssen, H. M.; Van As, H.; van Duynhoven, J. P. M. Scaling Behavior of Dendritic Nanoparticle Mobility in Semidilute Polymer Solutions. Macromolecules 2015, 48 (20), 7585-7591.

(3) Wang, J.; Yang, Y.; Yu, M.; Hu, G.; Gan, Y.; Gao, H.; Shi, X. Diffusion of Rod-like Nanoparticles in Non-adhesive and Adhesive Porous Polymeric Gels. J. Mech. Phys. Solids 2018, 112, 431-457.

(4) Skaug, M. J.; Wang, L.; Ding, Y.; Schwartz, D. K. Hindered Nanoparticle Diffusion and Void Accessibility in a Three-Dimensional Porous Medium. ACS Nano 2015, 9 (2), 2148-2156.

(5) Guan, J.; Wang, B.; Granick, S. Even Hard-Sphere Colloidal Suspensions Display Fickian Yet Non-Gaussian Diffusio. ACS Nano 2014, 8 (4), 3331-3336.

(6) He, K.; Babaye Khorasani, F.; Retterer, S. T.; Thomas, D. K.; Conrad, J. C.; Krishnamoorti, R. Diffusive Dynamics of Nanoparticles in Arrays of Nanoposts. ACS Nano 2013, 7 (6), 5122-5130.

(7) Yang, F.; Qian, S.; Zhao, Y.; Qiao, R. Self-Diffusiophoresis of Janus Catalytic Micromotors in Confined Geometries. Langmuir 2016, 32 (22), 5580-5592.

(8) Yang, L.; Zhang, Q.; Cui, Z.; Gerboth, M.; Zhao, Y.; Xu, T. T.; Walker, D. G.; Li, D. Ballistic Phonon Penetration Depth in Amorphous Silicon Dioxide. Nano Lett. 2017, 17 (12), 7218-7225.

(9) Jeon, J.-H.; Javanainen, M.; Martinez-Seara, H.; Metzler, R.; Vattulainen, I. Protein Crowding in Lipid Bilayers Gives Rise to NonGaussian Anomalous Lateral Diffusion of Phospholipids and Proteins. Phys. Rev. X 2016, 6 (2), 021006.

(10) Bronstein, I.; Israel, Y.; Kepten, E.; Mai, S.; Shav-Tal, Y.; Barkai, E.; Garini, Y. Transient Anomalous Diffusion of Telomeres in the Nucleus of Mammalian Cells. Phys. Rev. Lett. 2009, 103 (1), 018102.

(11) Wong, I. Y.; Gardel, M. L.; Reichman, D. R.; Weeks, E. R.; Valentine, M. T.; Bausch, A. R.; Weitz, D. A. Anomalous Diffusion
Probes Microstructure Dynamics of Entangled F-Actin Networks. Phys. Rev. Lett. 2004, 92 (17), 178101.

(12) Kalwarczyk, T.; Ziebacz, N.; Bielejewska, A.; Zaboklicka, E.; Koynov, K.; Szymanski, J.; Wilk, A.; Patkowski, A.; Gapinski, J.; Butt, H. J.; Holyst, R. Comparative Analysis of Viscosity of Complex Liquids and Cytoplasm of Mammalian Cells at the Nanoscale. Nano Lett. 2011, 11 (5), 2157-2163.

(13) Yu, M.; Wang, J.; Yang, Y.; Zhu, C.; Su, Q.; Guo, S.; Sun, J.; Gan, Y.; Shi, X.; Gao, H. Rotation-facilitated Rapid Transport of Nanorods in Mucosal Tissues. Nano Lett. 2016, 16 (11), 7176-7182.

(14) Chen, P.; Huang, Z.; Liang, J.; Cui, T.; Zhang, X.; Miao, B.; Yan, L.-T. Diffusion and Directionality of Charged Nanoparticles on Lipid Bilayer Membrane. ACS Nano 2016, 10 (12), 11541-11547.

(15) Lane, L. A.; Qian, X.; Smith, A. M.; Nie, S. Physical Chemistry of Nanomedicine: Understanding the Complex Behaviors of Nanoparticles in Vivo. Annu. Rev. Phys. Chem. 2015, 66, 521-547.

(16) Zhang, Y.; Dudko, O. K. First-Passage Processes in the Genome. Annu. Rev. Biophys. 2016, 45, 117-134.

(17) Song, X.; Feng, L.; Liang, C.; Yang, K.; Liu, Z. Ultrasound Triggered Tumor Oxygenation with Oxygen-Shuttle Nanoperfluorocarbon to Overcome Hypoxia-Associated Resistance in Cancer Therapies. Nano Lett. 2016, 16 (10), 6145-6153.

(18) Hofling, F.; Franosch, T. Anomalous transport in the crowded world of biological cells. Rep. Prog. Phys. 2013, 76, 046602.

(19) Wang, B.; Anthony, S. M.; Bae, S. C.; Granick, S. Anomalous yet Brownian. Proc. Natl. Acad. Sci. U. S. A. 2009, 106 (36), 1516015164.

(20) Wang, B.; Kuo, J.; Bae, S. C.; Granick, S. When Brownian diffusion is not Gaussian. Nat. Mater. 2012, 11, 481-485.

(21) Xue, C.; Zheng, X.; Chen, K.; Tian, Y.; Hu, G. Probing NonGaussianity in Confined Diffusion of Nanoparticles. J. Phys. Chem. Lett. 2016, 7 (3), 514-519.

(22) Kim, J.; Kim, C.; Sung, B. J. Simulation study of seemingly Fickian but heterogeneous dynamics of two dimensional colloids. Phys. Rev. Lett. 2013, 110 (4), 047801.

(23) Ge, T.; Grest, G. S.; Rubinstein, M. Nanorheology of Entangled Polymer Melts. Phys. Rev. Lett. 2018, 120 (5), 057801.

(24) English, B. P.; Hauryliuk, V.; Sanamrad, A.; Tankov, S.; Dekker, N. H.; Elf, J. Single-molecule investigations of the stringent response machinery in living bacterial cells. Proc. Natl. Acad. Sci. U. S. A. 2011, 108 (31), E365-373.

(25) He, W.; Song, H.; Su, Y.; Geng, L.; Ackerson, B. J.; Peng, H. B.; Tong, P. Dynamic heterogeneity and non-Gaussian statistics for acetylcholine receptors on live cell membrane. Nat. Commun. 2016, 7, 11701.

(26) Cai, L. H.; Panyukov, S.; Rubinstein, M. Hopping diffusion of nanoparticles in polymer matrices. Macromolecules 2015, 48 (3), 847862.

(27) Lungova, M.; Krutyeva, M.; Pyckhout-Hintzen, W.; Wischnewski, A.; Monkenbusch, M.; Allgaier, J.; Ohl, M.; Sharp, M.; Richter, D. Nanoscale Motion of Soft Nanoparticles in Unentangled and Entangled Polymer Matrices. Phys. Rev. Lett. 2016, 117 (14), 147803.

(28) Kalathi, J. T.; Yamamoto, U.; Schweizer, K. S.; Grest, G. S.; Kumar, S. K. Nanoparticle diffusion in polymer nanocomposites. Phys. Rev. Lett. 2014, 112 (10), 108301.

(29) Nahali, N.; Rosa, A. Nanoprobe diffusion in entangled polymer solutions: Linear vs. unconcatenated ring chains. J. Chem. Phys. 2018, 148 (19), 194902.

(30) Ghosh, S. K.; Cherstvy, A. G.; Grebenkov, D. S.; Metzler, R. Anomalous, non-Gaussian tracer diffusion in crowded two-dimensional environments. New J. Phys. 2016, 18 (1), 013027.

(31) Kim, Y. J.; Choi, J. K.; Lee, D.-G.; Baek, K.; Oh, S. H.; Lee, I. S. Solid-State Conversion Chemistry of Multicomponent Nanocrystals Cast in a Hollow Silica Nanosphere: Morphology-Controlled Syntheses of Hybrid Nanocrystals. ACS Nano 2015, 9 (11), 10719-10728. 
(32) Rouxel, T. Thermodynamics of viscous flow and elasticity of glass forming liquids in the glass transition range. J. Chem. Phys. 2011, 135 (18), 184501.

(33) Meng, D.; Zhang, K.; Kumar, S. K. Size-dependent penetrant diffusion in polymer glasses. Soft Matter 2018, 14 (21), 4226-4230.

(34) Rubinstein, M.; Colby, R. H. Polymer Physics; Oxford University Press: New York, 2003.

(35) Lee, C. H.; Crosby, A. J.; Emrick, T.; Hayward, R. C. Characterization of Heterogeneous Polyacrylamide Hydrogels by Tracking of Single Quantum Dots. Macromolecules 2014, 47 (2), 741-749.

(36) van Zanten, J. H.; Amin, S.; Abdala, A. A. Brownian motion of colloidal spheres in aqueous PEO solutions. Macromolecules 2004, 37, 3874-3880.

(37) Poling-Skutvik, R.; Krishnamoorti, R.; Conrad, J. C. SizeDependent Dynamics of Nanoparticles in Unentangled Polyelectrolyte Solutions. ACS Macro Lett. 2015, 4 (10), 1169-1173.

(38) Cai, L. H.; Panyukov, S.; Rubinstein, M. Mobility of nonsticky nanoparticles in polymer liquids. Macromolecules 2011, 44, 78537863.

(39) Brochard Wyart, F.; de Gennes, P. G. Viscosity at small scales in polymer melts. Eur. Phys. J. E: Soft Matter Biol. Phys. 2000, 1 (1), 93-97.

(40) Phillies, G. D. J. The hydrodynamic scaling model for polymer self-diffusion. J. Phys. Chem. 1989, 93, 5029-5039.

(41) Chubynsky, M. V.; Slater, G. W. Diffusing Diffusivity: A Model for Anomalous, yet Brownian, Diffusion. Phys. Rev. Lett. 2014, 113 (9), 098302.

(42) Ernst, D.; Kohler, J.; Weiss, M. Probing the type of anomalous diffusion with single-particle tracking. Phys. Chem. Chem. Phys. 2014, 16 (17), 7686-7691.

(43) Samanta, N.; Chakrabarti, R. Tracer diffusion in a sea of polymers with binding zones: mobile vs. frozen traps. Soft Matter 2016, 12 (41), 8554-8563.

(44) Dell, Z. E.; Schweizer, K. S. Theory of localization and activated hopping of nanoparticles in cross-linked networks and entangled polymer melts. Macromolecules 2014, 47 (1), 405-414.

(45) Nath, P.; Mangal, R.; Kohle, F.; Choudhury, S.; Narayanan, S.; Wiesner, U.; Archer, L. A. Dynamics of Nanoparticles in Entangled Polymer Solutions. Langmuir 2018, 34 (1), 241-249.

(46) Chaudhuri, P.; Berthier, L.; Kob, W. Universal nature of particle displacements close to glass and jamming transitions. Phys. Rev. Lett. 2007, 99 (6), 060604.

(47) Sentjabrskaja, T.; Zaccarelli, E.; De Michele, C.; Sciortino, F.; Tartaglia, P.; Voigtmann, T.; Egelhaaf, S. U.; Laurati, M. Anomalous dynamics of intruders in a crowded environment of mobile obstacles. Nat. Commun. 2016, 7, 11133.

(48) Shi, J.; Kantoff, P. W.; Wooster, R.; Farokhzad, O. C. Cancer nanomedicine: progress, challenges and opportunities. Nat. Rev. Cancer 2017, 17 (1), 20-37.

(49) Blanco, E.; Shen, H.; Ferrari, M. Principles of nanoparticle design for overcoming biological barriers to drug delivery. Nat. Biotechnol. 2015, 33 (9), 941-951.

(50) Wagner, C. E.; Turner, B. S.; Rubinstein, M.; McKinley, G. H.; Ribbeck, K. A Rheological Study of the Association and Dynamics of MUC5AC Gels. Biomacromolecules 2017, 18 (11), 3654-3664.

(51) Thapa, S.; Lomholt, M. A.; Krog, J.; Cherstvy, A. G.; Metzler, R. Bayesian analysis of single-particle tracking data using the nestedsampling algorithm: maximum-likelihood model selection applied to stochastic-diffusivity data. Phys. Chem. Chem. Phys. 2018, 20 (46), 29018-29037.

(52) Li, Z.; Di, C.; Li, S.; Yang, X.; Nie, G. Smart Nanotherapeutic Targeting of Tumor Vasculature. Acc. Chem. Res. 2019, 52 (9), 27032712.

(53) Sun, H.; Wang, Z.; He, Y. Direct Observation of Spatiotemporal Heterogeneous Gelation by Rotational Tracking of a Single Anisotropic Nanoprobe. ACS Nano 2019, 13 (10), 1133411342 .
(54) Yu, M.; Song, W.; Tian, F.; Dai, Z.; Zhu, Q.; Ahmad, E.; Guo, S.; Zhu, C.; Zhong, H.; Yuan, Y.; Zhang, T.; Yi, X.; Shi, X.; Gan, Y.; Gao, H. Temperature- and rigidity-mediated rapid transport of lipid nanovesicles in hydrogels. Proc. Natl. Acad. Sci. U. S. A. 2019, 116 (12), 5362-5369.

(55) Balk, A. L.; Mair, L. O.; Mathai, P. P.; Patrone, P. N.; Wang, W.; Ahmed, S.; Mallouk, T. E.; Liddle, J. A.; Stavis, S. M. Kilohertz Rotation of Nanorods Propelled by Ultrasound, Traced by Microvortex Advection of Nanoparticles. ACS Nano 2014, 8 (8), 83008309. 